\title{
Three-body force effect on the properties of neutron-rich nuclear matter
}

\author{
Wei Zuo ${ }^{1}$, Ignazio Bombarci ${ }^{2}$ and Umberto Lombardo ${ }^{3}$
}

${ }^{1}$ Institute of Modern Physics, Chinese Academy of Sciences, Lanzhou 730000, China

and State Key Laboratory of Theoretical Physics, Institute of Theoretical

Physics, Chinese Academy of Sciences, Beijing 100190, China

2 Dipartimento di Fisica "E. Fermi", Università di Pisa, and INFN, Sezione di Pisa, Largo B. Pontecorvo, 3 I-56127 Pisa, Italy

3 Università di Catania and INFN - Laboratori Nazionali del Sud, Catania 95123, Italy

\begin{abstract}
We review our research work in recent years on the properties of neutron-rich nuclear matter within the framework of the Brueckner theory extended to include a microscopic three-body force (TBF). We discuss specially the TBF effect on the equation of state and the singleparticle (s.p.) potentials. The TBF turns out to be crucial for describing the saturation properties of nuclear matter in nonrelativistic microscopic framework. The TBF effect on the EOS of neutron-rich nuclear matter is to result in a significant stiffening of its isovector part at supra-saturation densities. Within the Brueckner approach, the TBF may lead to a rearrangement contribution to the s.p. potentials, which enhances strongly the repulsion and momentum-dependence of the s.p. potentials at high densities and high momenta. Our results are also compared with the predictions by other $a b$ initio approaches.
\end{abstract}

The equation of state (EOS) and single-particle properties of neutronrich nuclear matter play an important role in many issues of nuclear physics and nuclear astrophysics, including the structure and collective properties of heavy nuclei and neutron-rich nuclei [1-7]. The isovector part of the EOS 
of neutron-rich nuclear matter is determined by nuclear symmetry energy. At densities around and below nuclear saturation density, symmetry energy is not only closely related to the structure of neutron-rich nuclei away from the nuclear stability line [8], it is also shown to be decisive for explaining the experimentally observed enhancement of the stability against $\alpha$-decay with increasing the mass number along an isotope chain for the synthesized superheavy nuclei not around shell closures [9]. At high-densities well above the normal density, the density dependence of symmetry energy is expected to be extremely important for modeling the structure and cooling properties of neutron stars.

Theoretically, the properties of neutron-rich nuclear matter can be predicted within various nuclear many-body frameworks, including both phenomenological and microscopic approaches. In the phenomenological methods, the parameters of the effective interactions are essentially obtained by reproducing the ground state properties of stable nuclei and/or the saturation properties of nuclear matter, and the predicted high-density behavior using different parameter sets may be largely different and even be opposite [10]. Within the ab initio approaches, the properties of neutron-rich nuclear matter have been investigated extensively in recent years. Almost all the $a b$ initio predictions indicate an monotonically increasing symmetry energy as a function of density, whereas the stiffness of the density dependence predicted by adopting different approaches and/or different NN interactions turns out to be largely different at high densities. In our investigation, we use the Breuckner-Hartree-Fock (BHF) method based on the BBG theory [11]. The extension of the BBG scheme to asymmetric nuclear matter and to include microscopic three-body forces can be found in Refs. [12,13] and Refs. [14-16], respectively.

As well known, a nonrelativistic many-body approach of rigid nucleons interacting via realistic two-body forces fitting in-vacuum nucleon-nucleon scattering data can not reproduce the empirical saturation properties of nuclear mattery [17]. To solve this problem, one has to introduce threebody forces or to adopt a relativistic theory, such as Dirac-BHF method. Up to now, several different kinds of TBF model have been applied in the BHF calculations. One is the semiphenomenological TBF model, such as the Urbana TBF, in which the adjustable parameters are usually determined by fitting the observed triton binding energies and/or the empirical saturation properties of symmetric nuclear matter. Another kind of TBF model adopted in the Brueckner theory is the microscopic one $[15,16,18]$ based on the meson exchange theory for NN interactions. There is no adjustable parameters in the sense that the meson parameters are essentially 


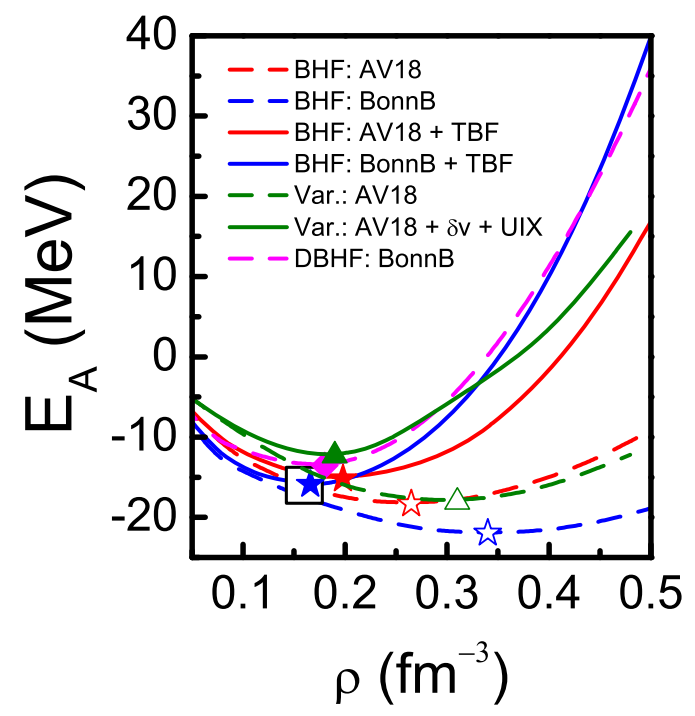

Figure 1: EOS of symmetric nuclear matter predicted by different microscopic approaches. The results of the BHF approach are taken from Refs. [16] and [18]. The Dirac-BHF prediction is taken from Ref. [19]. The results by the variational approach are from Ref. [20].

determined self-consistently by the corresponding two-body force. The extension to include the $\sigma$ and $\omega$ exchanges as well as the associated virtual nucleon-antinucleon pair excitations and nucleon resonances have been done by Grange et al. [15]. Further improvement and development of the model have been achieved in Refs. [14,16,18]. In our BHF calculation, the microscopic TBFs have been adopted and the TBF contribution is included by reducing the TBF to an equivalently effective two-body interaction according to the standard and extensively adopted scheme [15].

In Fig. 1, we show the TBF effect on the EOS of symmetric nuclear matter. In the figure, the predictions from different $a b$ initio theoretical frameworks are compared, including the BHF approach [16,18], the manybody variational method [20], and the Dirac-BHF theory [19]. It is clear that the TBF provides a repulsive contribution to the nuclear EOS in both the nonrelativistic BHF and variational approaches, and the TBF repulsion increases monotonically as a function of density. Inclusion of the TBF contribution turns out to be necessary for reproducing the empirical satura- 
tion property of nuclear matter in a non-relativistic microscopic framework. Within the BHF framework, the predicted saturation densities are approximately $0.265 \mathrm{fm}^{-3}$ and $0.33 \mathrm{fm}^{-3}$ by using the AV18 and BonnB two-body interactions, respectively, which are much larger than the empirical value and the Dirac-BHF prediction of roughly $0.18 \mathrm{fm}^{-3}$. By including the corresponding TBFs, the saturation densities are improved remarkably to about $0.167 \mathrm{fm}^{-3}$ and $0.19 \mathrm{fm}^{-3}$.

In comparison with the Dirac-BHF theory, it has been shown $[16,18]$ quantitatively that the main relativistic correction to the EOS of nuclear matter can be reproduced within the nonrelativistic BHF framework by the TBF component involving the virtual excitations of nucleon-antinucleon pairs due to the $2 \sigma$-meson exchange.

As for neutron-rich nuclear matter, the energy per nucleon has been shown to fulfil satisfactorily a linear dependence on $\beta^{2}$ in the whole asymmetry range of $0 \leq \beta \leq 1$ by various ab initio investigations by adopting different realistic NN interactions, i.e., $E_{A}(\rho, \beta)=E_{A}(\rho, 0)+E_{\text {sym }}(\rho) \beta^{2}$. Inclusion of the TBF effect does not destroy the linear dependence of $E_{A}(\rho, \beta)$ on $\beta^{2}[16]$. The simple $\beta^{2}$-law implies that the isospin dependence of the EOS of neutron-rich nuclear matter is governed completely by the symmetry energy. Due to the linear dependence of $E_{A}(\rho, \beta)$ on $\beta^{2}$, the symmetry energy can be calculated directly by the difference between the EOS of pure neutron matter and that of symmetric nuclear matter, i.e., $E_{\text {sym }}(\rho)=E_{A}(\rho, 1)-E_{A}(\rho, 0)$. Another important consequence of the $\beta^{2}$-law is that the difference of the neutron and proton chemical potentials in neutron star matter can be related to the symmetry energy in a explicitly way: $\mu_{n}-\mu_{p}=4 \beta E_{\text {sym }}$.

To see clearly the TBF effect on the EOS of neutron-rich nuclear matter, we display, in Fig. 2, the density dependence of symmetry energy predicted within various ab initio frameworks, including the BHF approach $[16,18]$, the variational methed [20], and the Dirac-BHF theory [21]. At low densities around and below the saturation density, the TBF effect turns out to be reasonably weak, and the difference among the different $a b$ initio predictions is seen to be quite small. It is worth noticing that the symmetry energy predicted by the three different $a b$ initio approaches and/or using different realistic NN interactions increases monotonically as a function of density. However, the density dependence of symmetry energy from different approaches may become quite different at high densities. The TBF effect on symmetry energy is repulsive. The TBF repulsion increases rapidly at supra-saturation density, and leads to a stiffening of the density dependence of symmetry energy at high densities. It is worth noticing that the TBF effect on symmetry energy is much stronger at high densities within the 


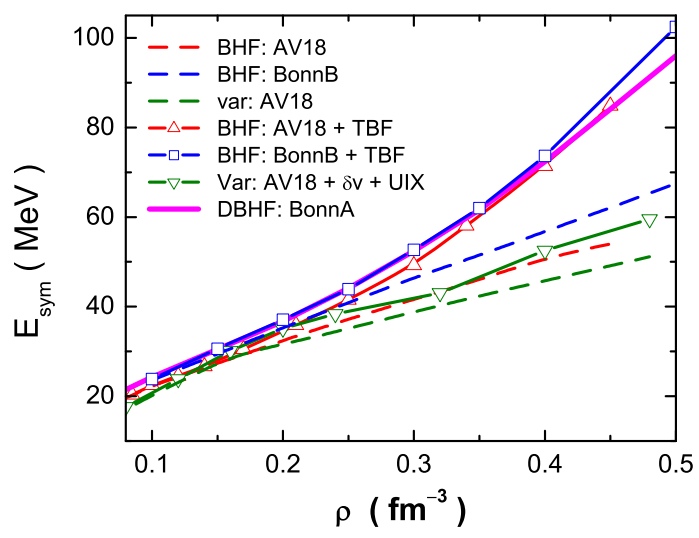

Figure 2: Density dependence of symmetry energy predicted within three different $a b$ initio theoretical frameworks. The results of the BHF approach are from Refs. [16] and [18]. The Dirac-BHF prediction is taken from Ref. [21]. The results by the variational approach are from Ref. [20].

BHF framework than that within the variational framework. Inclusion of the TBFs in the BHF calculations makes the predicted symmetry energy almost completely coincide with the Dirac-BHF prediction up to $\rho=0.5 \mathrm{fm}^{-3}$. Whereas, the symmetry energy obtained within the variational approach using the AV18 interaction plus the UIX TBF and the boost correction remains far away from the Dirac-BHF result at high densities well above the saturation density.

At the lowest mean field approximation, there exist two problems in the BHF approach for predicting nuclear s.p. properties. First, at densities around the saturation density, the predicted optical potential depth is too deep as compared to the empirical value, and it destroy the HugenholtzVan Hove (HVH) theorem. This problem essentially by including the effect of ground state correlations in calculating the s.p. properties. Second, at high densities, the predicted potential is too attractive and its momentum dependence turns out to be too weak at the lowest-order BHF approach for describing the experimental elliptic flow data [22]. Concerning these two problems, we have improved the BHF calculation of the s.p. properties inn two aspects. One is to extend the calculation of the effect of ground state correlations to neutron-rich nuclear matter. The second is to take into account the TBF-induced rearrangement contribution for calculating 
the s.p. properties within the Brueckner theory.

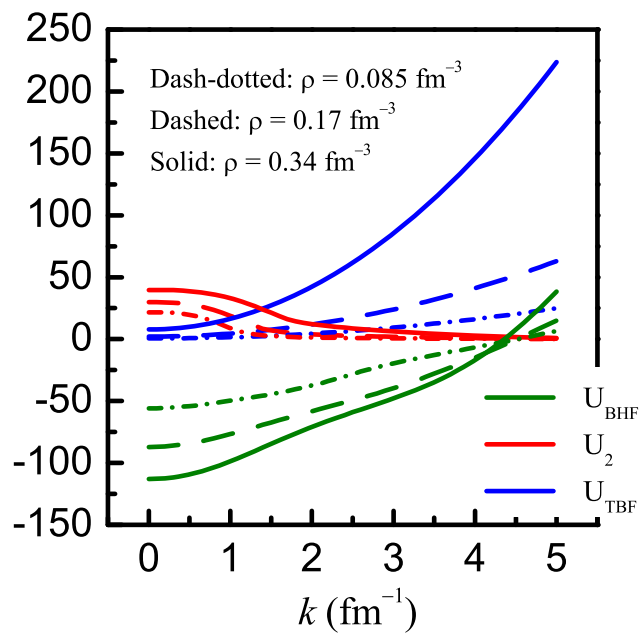

Figure 3: The three different contributions $U_{\mathrm{BHF}}, U_{2}$, and $U_{\mathrm{TBF}}$ in symmetric nuclear matter at three densities. Taken from Ref. [23].

In Fig. 3, we show separately the three different contributions (i.e., the BHF s.p. potential $U_{\mathrm{BHF}}$, the Pauli rearrangement term $U_{2}$, and the TBF rearrangement contribution $U_{\mathrm{TBF}}$ ) in symmetric nuclear matter at there typical densities of $\rho=0.085,0.17$, and $0.34 \mathrm{fm}^{-3}$. The BHF s.p. potential is strongly attractive at low momenta and its attraction decreases as a function of momentum. The g.s. correlations affect the s.p. potential mainly at low momenta, and lead to a repulsive contribution $U_{2}$. which is considerably smaller in magnitude than the lowest-order BHF contribution $U_{\mathrm{BHF}}$. The contribution of g.s. correlations decreases rapidly around the Fermi mumentum and vanishes at high momenta well above the Fermi momentum. The TBF induces a strongly repulsive and momentum-dependent rearrangement contribution to the nucleon s.p. potential, at high densities and momenta in nuclear medium, which has been shown [24,25] to be crucial for improving the large-density and high-momentum behavior of the BHF s.p. potential. For a detailed discussion about the isospin-dependence of the three different contributions in neutron-rich nuclear matter, we refer to our review paper [1]. 
In summary, We have reported briefly our research work on the properties of neutron-rich nuclear matter within the framework of the Brueckner theory. Special attention has been paid to the discussion of the TBF effect and the comparison of our results with the predictions by other ab initio approaches. It is shown that the TBF effect provides a repulsive contribution to the EOS of nuclear matter and improves remarkably the predicted saturation properties within a nonrelativistic ab initio approach. Inclusion of the TBF does not destroy the empirical parabolic law fulfilled by the EOS of neutron-rich nuclear matter. The TBF may lead to a strong enhancement of the stiffness of symmetry energy at high densities. In predicting the s.p. properties in neutron-rich nuclear matter, we have improved the Brueckner calculation in two aspects. One is to extend the calculation of the g.s. correlation effect to neutron-rich nuclear matter; the second is to include the rearrangement contribution induced by the TBF. The TBFinduced rearrangement contribution turns out to be strongly repulsive and momentum-dependent at high densities and momenta, which is necessary for improving the large-density and high-momentum behavior of the s.p. potentials.

The work is partly supported by the National Natural Science Foundation of China $(11435014,11175219)$, the 973 Program of China (Grant No. 2007CB815004), and the Knowledge Innovation Project(KJCX2-EW-N01) of the Chinese Academy of Sciences.

\section{References}

[1] Wei Zuo, Ignazio Bombaci, and Umberto Lombardo, Eur. Phys. J. A 50 (2014) 12 and reference therein.

[2] B. A. Li, L. C. Chen and C. M. Ko, Phys. Rep. 464, (2008) 113

[3] P. Danielewicz, R. Lacey and W. G. Lynch, Science 298, (2002) 1592.

[4] W. Zuo, A. Li, Z. H. Li and U. Lombardo, Phys. Rev. C 70, (2004) 055802 .

[5] M. Baldo and C. Maieron, J. Phys. G 34, (2007) R243.

[6] C. Fuchs and H. H. Wolter, Eur. Phys. J. A 30, (2006) 5.

[7] V. Barana, M. Colonna, V. Grecoc and M. Di Toro, Phys. Rep. 410, (2005) 335. 
[8] J. M. Dong, W. Zuo, J. Z. Gu, and U. Lombardo, Phys. Rev. C 85, (2012) 034308.

[9] J. M. Dong. W. Zuo and W. Scheid, Phys. Rev. Lett. 107, (2011) 012501.

[10] L. W. Chen, C. M. Ko and B. A. Li, Phys. Rev C 72, (2005) 064309.

[11] B. D. Day, Rev. Mod. Phys. 39, (1967) 719.

[12] I. Bombaci, U. Lombardo, Phys. Rev. C 44, (1991) 1892.

[13] W. Zuo, I. Bombaci, and U. Lombardo, Phys. Rev. C 60, (1999) 024605.

[14] W. Zuo, A. Lejeune, U. Lombardo and J. F. Mathiot, Eur. Phys. J. A 14, (2002) 469.

[15] P. Grangé, A. Lejeune, M. Martzolff, and J. F. Mathiot, Phys. Rev. C 40, (1989) 1040.

[16] W. Zuo, A. Lejeune, U. Lombardo and J. F. Mathiot, Nucl. Phys. A 706, (2002) 418.

[17] Z. H. Li, U. Lombardo, H. J. Schulze, W. Zuo, L. W. Chen and H. R. Ma, Phys. Rev. C 74, (2006) 047304.

[18] Z. H. Li, U. Lombardo, H. J. Schulze and W. Zuo, Phys. Rev. C 77, (2008) 034316.

[19] R. Brockmann and R. Machleidt, Phys. Rev. C 42, (1990) 1965.

[20] A. Akmal, V. R. Pandharipande, and D. G. Ravenhall, Phys. Rev. C 58, (1998) 1804.

[21] E. N. E. van Dalen, C. Fuchs and A. Faessler, Nucl.Phys. A 744, (2004) 227; Phys. Rev. C 72, (2005) 065803.

[22] P. Danielewicz, Nucl. Phys. A 673, (2000) 375.

[23] W. Zuo, Nucl. Phys. A 834, (2010) 574c.

[24] W. Zuo, L. G. Cao, B. A. Li, U. Lombardo and C. W. Shen Phys. Rev. C 72 (2005) 014005.

[25] W. Zuo, U. Lombardo, H. J. Schulze and Z. H. Li, Phys. Rev. C 74, (2006) 014317. 